\title{
The early transcriptomic response to interleukin $1 \beta$ and interleukin 33 in rat neonatal cardiomyocytes
}

Article

Accepted Version

Barrett, O. P. T., Yndestad, A., Marshall, A. K., Sugden, P. H. and Clerk, A. (2013) The early transcriptomic response to interleukin $1 \beta$ and interleukin 33 in rat neonatal cardiomyocytes. Cytokine, 61 (2). pp. 340-344. ISSN 10960023 doi: https://doi.org/10.1016/j.cyto.2012.11.004 Available at https://centaur.reading.ac.uk/30656/

It is advisable to refer to the publisher's version if you intend to cite from the work. See Guidance on citing.

Published version at: http://dx.doi.org/10.1016/j.cyto.2012.11.004

To link to this article DOI: http://dx.doi.org/10.1016/j.cyto.2012.11.004

Publisher: Elsevier

All outputs in CentAUR are protected by Intellectual Property Rights law, including copyright law. Copyright and IPR is retained by the creators or other copyright holders. Terms and conditions for use of this material are defined in the End User Agreement.

www.reading.ac.uk/centaur 
Central Archive at the University of Reading

Reading's research outputs online 
The early transcriptomic response to interleukin $1 \beta$ and interleukin 33 in rat neonatal cardiomyocytes

Oliver P.T. Barrett ${ }^{\mathrm{a}}$, Arne Yndestad ${ }^{\mathrm{b}}$, Andrew K. Marshall ${ }^{\mathrm{c}}$, Peter H. Sugden ${ }^{\mathrm{c}}$, Angela Clerk $^{\mathrm{c}^{*}}$.

${ }^{a}$ Department of Life Sciences, Imperial College London, London, UK

${ }^{\mathrm{b}}$ Research Institute for Internal Medicine, Oslo University Hospital Rikshospitalet, Oslo, Norway

${ }^{c}$ School of Biological Sciences, University of Reading, Reading, UK

* Corresponding author at: School of Biological Sciences, Harborne Building, University of Reading, Whiteknights, Reading RG6 6BX, UK. Tel.: +44 118378 8932. E-mail address: a.clerk@ reading.ac.uk (A.Clerk). 


\begin{abstract}
In the heart, inflammatory cytokines including interleukin (IL) $1 \beta$ are implicated in regulating adaptive and maladaptive changes, whereas IL33 negatively regulates cardiomyocyte hypertrophy and promotes cardioprotection. These agonists signal through a common coreceptor but, in cardiomyocytes, IL1 $\beta$ more potently activates mitogen-activated protein kinases and $\mathrm{NF \kappa B}$, pathways that regulate gene expression. We compared the effects of external application of IL1 $\beta$ and IL33 on the cardiomyocyte transcriptome. Neonatal rat cardiomyocytes were exposed to IL1 $\beta$ or IL33 $(0.5,1$ or $2 \mathrm{~h})$. Transcriptomic profiles were determined using Affymetrix rat genome 2302.0 microarrays and data were validated by quantitative PCR. IL1 $\beta$ induced significant changes in more RNAs than IL33 and, generally, to a greater degree. It also had a significantly greater effect in downregulating mRNAs and in regulating mRNAs associated with selected pathways. IL33 had a greater effect on a small, select group of specific transcripts. Thus, differences in intensity of intracellular signals can deliver qualitatively different responses. Quantitatively different responses in production of receptor agonists and transcription factors may contribute to qualitative differences at later times resulting in different phenotypic cellular responses.
\end{abstract}

Keywords: Interleukins, cardiomyocytes, microarrays, mitogen-activated protein kinases, RNA expression

Abbreviations: ERK, extracellular signal-regulated kinase; IL, interleukin; MAPK, mitogenactivated protein kinase 


\section{Introduction}

The interleukin 1 (IL1) family cytokines include IL1 $\alpha / \beta$ which bind to IL1R1, and IL33 which binds to a transmembrane form of ST2 (gene symbol, IL1RL1) [1]. IL1R1 and ST2 interact with the same co-receptors, IL1R accessory proteins (IL1RAcPs), forming similar ternary complexes [2]. IL1RAcPs are the signaling components, recruiting adapter molecules (MyD88, IRAKs, TRAF6) that stimulate intracellular signaling pathways including mitogen-activated protein kinases (MAPKs) [extracellular signal-regulated kinases

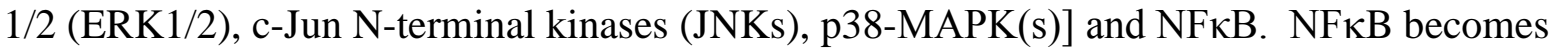
nuclear-localised where it stimulates transcription directly, whereas MAPKs regulate mRNA expression through phosphorylation of transcription factors to modulate their transactivating activities or via proteins that regulate mRNA stability [3].

IL1 $\beta$ is a major component of the innate immune response and is immensely important as an upstream initiator of inflammation [4]. IL33 is implicated in various systems, playing a particular role in initiating Th2-type responses (e.g. asthma and arthritis [5]). In heart, inflammatory cytokines including IL1 $\beta$ are associated with regulation of both adaptive and maladaptive changes [6] whereas IL33, despite having inflammatory effects, negatively regulates cardiomyocyte hypertrophy and promotes cardioprotection [7]. In many cells, IL33 is nuclear-localized and is released during apoptosis to initiate an inflammatory response [5, 8]. Alternatively, in fibroblasts, IL33 release may be instigated by mechanical strain [9]. Since cardiac fibroblasts are the major source of IL33 in the heart [10], increased biomechanical stretch/strain potentially stimulates release of IL33 from fibroblasts for paracrine stimulation of adjacent cardiomyocytes. Differences in receptor-based signaling alone could account for the differing effects of IL1 $\beta$ vs IL33 on cardiomyocytes and the heart. Although IL1 $\beta$ and IL33 stimulate similar temporal activation of MAPKs in cardiomyocytes 
(consistent with signaling through the same co-receptor), the degree of activation of these pathways and NFKB by IL1 $\beta$ is much greater than that induced by IL33 [10-12]. This probably reflects relative levels of expression of ligand binding receptors. Nevertheless, differential regulation of the same canonical signaling pathway can elicit different phenotypic outcomes. This is most well-established in PC12 cells in which differential regulation of ERK1/2 results in proliferation or differentiation [13]. A similar scenario may operate in cardiomyocytes exposed to IL1 $\beta$ vs IL33. Here, we used RNA expression profiling as a measure of the output from IL1 $\beta$ and IL33 signaling in cardiomyocytes. We demonstrate qualitative (rather than purely quantitative) differences in the profiles, propagation of which could contribute to the different effects of these cytokines on cardiac responses.

\section{Methods}

Neonatal rat ventricular cardiomyocytes were prepared from 2-4 day Sprague-Dawley rats as described [14]. Animals (maintained in accordance with Directive 2010/63/EU of the European Parliament) were culled by cervical dislocation. Cells were plated at $4 \times 10^{6}$ cells per $60 \mathrm{~mm}$ Primaria ${ }^{\mathrm{TM}}$ tissue culture dish in serum-containing medium $(18 \mathrm{~h})$. Cells were then cultured in serum-free medium ( $24 \mathrm{~h}$ ) before addition of agonist. Recombinant rat IL1 $\beta$ (R \& D Systems) and recombinant rat IL33 (Axxora) were prepared and diluted in PBS containing $0.1 \%(\mathrm{v} / \mathrm{v}) \mathrm{BSA}$.

Cardiomyocytes were unstimulated or exposed to IL1 $\beta$ or IL33 and total RNA prepared as previously described [14]. To minimise variation resulting from different cardiomyocyte preparations, equal amounts of RNA from three individual experiments were pooled to generate a single sample set. Samples ( 3 sets) were prepared and hybridized to Affymetrix rat genome 2302.0 arrays as previously described [14]. The data are available through ArrayExpress (ArrayExpress ID: E-MEXP-1960 and E-MEXP-1912). Files (.CEL) 
were imported into GeneSpring 11.5 (Agilent Technologies) and data were normalized using MAS5. Normalisation per gene was to the corresponding controls within each sample set. Probesets were selected for analysis if present or marginal in all of any condition, and filtered according to fold change ( $>1.5$-fold) relative to controls. Probesets with statistically significant changes (false discovery rate, FDR $<0.05$ ) were identified by one-way ANOVA with Student-Newman-Keuls (SNK) post-test, using the Benjamini and Hochberg multiple testing correction. Data for IL1 $\beta$ and IL33 were analysed separately and lists combined for downstream analysis. Pathway analysis used Panther 7.2 and genes were classified according to Panther profiles [15].

qPCR was performed using an ABI Real-Time PCR 7500 system (Applied Biosystems) as previously described $[11,14]$. Values for mRNAs (see Supplemental Table S1 for primer details) were normalized to glyceraldehyde 3-phosphate dehydrogenase (Gapdh) expression and then to control values.

\section{Results and discussion}

Neonatal rat ventricular myocytes were used for this study. As terminallydifferentiated cells, they are highly synchronised and they can be rendered quiescent by serum starvation. Freshly isolated adult cardiomyocytes are stressed by the isolation procedure (MAPKs are activated with baseline changes in IEG expression, unpublished data) and cultured adult cardiomyocytes undergo apoptosis (25-50\% loss of viability over $48 \mathrm{~h}$ ) with dedifferentiation of surviving cells over time $[16,17]$, so they are less suitable. Cardiomyocytes were exposed to $100 \mathrm{ng} / \mathrm{ml}$ IL1 $\beta$ or IL33 (since $100 \mathrm{ng} / \mathrm{ml}$ IL1 $\beta$ is required for maximal activation of JNKs and $100 \mathrm{ng} / \mathrm{ml}$ IL33 gives maximal activation of MAPKs 
without complications from sST2 [11, 12]), and we examined the early changes in RNA expression $(0.5,1$ and $2 \mathrm{~h}$ ) to focus on immediate early genes (IEGs). IEGs are regulated by pre-existing transcription factors immediately downstream of the protein kinase signaling events and are representative of receptor signaling.

Using Affymetrix microarrays, 609 RNAs were identified with significant changes in expression ( $>1.5$-fold, FDR<0.05) in cardiomyocytes exposed to IL1 $\beta$ or IL33 (Supplemental Spreadsheet S1). The majority were selectively regulated by IL1 $\beta$ (247 RNAs upregulated; 229 RNAs downregulated) (Fig. 1A) and, even for transcripts that responded to either agonist (91 RNAs upregulated; 8 RNAs downregulated), most exhibited a greater response to IL1 $\beta$ than IL33 (Fig. 1B). A few transcripts (19 RNAs upregulated; 15 RNAs downregulated) were significantly changed only in response to IL33 (Fig. 1C). We did not detect IL5 or IL13 in cardiomyocytes exposed to IL33, although they are upregulated by IL33 in other cells [18]. This may be a cell-specific difference and/or reflect our focus on the early response to IL33 stimulation (in contrast to other studies that emphasise longer term changes over, for example, $48 \mathrm{~h}[18])$.

To validate the microarray data by qPCR, we focused on inflammation-associated genes, selecting six that were upregulated by IL1 $\beta$ (Csf1, Csf2), both agonists (Csf3, Ptgs2) or IL33 (IL1 $\alpha$, IL1 $\beta$ ) and examining their regulation over a more detailed time course (Fig. 1, D - I). The effect of IL33 on expression of IL $1 \alpha / \beta$ mRNA suggests that some downstream, late effects of IL33 may be mediated via these cytokines. Other genes were chosen because of their association with cardiac disorders. Colony stimulating factor 1 (Csf1, also known as macrophage colony stimulating factor) is upregulated in heart in conditions of unstable angina and following myocardial infarction [19]. Csf2 (granulocyte and macrophage colony stimulating factor) and Csf3 (granulocyte colony stimulating factor) are both associated with cardiac remodelling following infarction [20]. Ptgs2 (also known as cyclooxygenase 2) 
influences cardiac function, though whether or not it is beneficial is debated [21]. For all mRNAs, qPCR (left panels) and microarray data (right panels) were in accord. IL1 $\beta$ had a substantially greater effect than IL33 on expression of Csf1 and Csf 2 mRNAs at all times studied (Fig. 1, D and E). Both agonists increased expression of Csf3 and Ptgs2 mRNAs, although IL1 $\beta$ still had a greater effect particularly at prolonged times (Fig. 1, F and G). IL33 (but not IL1 $\beta$ ) increased expression of IL1 $\alpha$ and IL1 $\beta$ mRNAs over 0.5 - 2 h (Fig. 1, H and I).

IL1 $\beta$-responsive RNAs were clustered according to the time for maximal upregulation (Fig. 2A) or maximal downregulation (Fig. 2B). Each cluster was then interrogated for IL33responsive RNAs (Supplemental Table S2). Cluster 1 (65 IL1 $\beta$-responsive RNAs with maximal upregulation at $0.5 \mathrm{~h})$ contained IL33-responsive RNAs with similar profiles $(\mathrm{n}=13)$ or with maximal upregulation at $1 \mathrm{~h}$ and a more sustained response $(\mathrm{n}=18)$. The degree of stimulation by IL33 was less than that induced by IL1 $\beta$. Cluster 2 (64 IL1 $\beta$-responsive RNAs with maximal upregulation at $1 \mathrm{~h}$ ) contained 19 IL33-responsive RNAs with similar profiles and degrees of stimulation. Cluster 3 (199 IL1 $\beta$-responsive RNAs with maximal upregulation at $2 \mathrm{~h}$ ) contained IL33-responsive RNAs with either similar profiles and degrees of upregulation $(n=51)$ or greater stimulation at $0.5-1 \mathrm{~h}(\mathrm{n}=13)$. Cluster 4 (12 IL1 $\beta$ responsive RNAs with maximal downregulation at $0.5 \mathrm{~h}$ ) contained no IL33-responsive RNAs. Cluster 5 (34 IL1 $\beta$-responsive RNAs with maximal downregulation at $1 \mathrm{~h}$ ) contained 4 IL33-responsive RNAs with maximal downregulation at $2 \mathrm{~h}$. Cluster 6 (195 IL1ßresponsive RNAs with maximal downregulation at $2 \mathrm{~h}$ ) contained 14 IL33 responsive RNAs with similar profiles and degrees of downregulation. We classified the genes according to the Panther system and profiles which considers the numbers in each category relative to the total number in the list and the total number in the database to generate a probability value for whether or not the pathway is over-represented [15]. Both agonists regulated genes 
associated with inflammation-related pathways (Fig. 2C; Supplemental Table S3). However, IL1 $\beta$ also significantly affected genes associated with platelet-derived growth factor signaling, with a greater effect than IL33 on interleukin signaling and angiogenesis.

In conclusion, our transcriptomics data indicate that the changes in RNA expression profiles induced by extracellular application of IL1 $\beta$ and IL33 (i.e. focusing only on receptormediated effects) are qualitatively and quantitatively different. Generally, IL1 $\beta$ induced a greater change in the cardiomyocyte transcriptome than IL33 in terms of numbers of RNAs and degree of change of individual RNAs. Probably the most striking difference was in the greater number of downregulated RNAs in response to IL1 $\beta$. The early qualitative differences could lead to different phenotypic outcomes in the heart. However, quantitative differences in regulation of downstream cytokines with the generation of different cytokine profiles are also likely to propagate a differential phenotypic outcome. Importantly, while a balanced inflammatory response as that induced by IL33 could induce tolerance and cardioprotection, excessive inflammation induced by IL1 $\beta$ could cause collateral damage and contribute to maladaptive changes in the heart.

\section{Acknowledgements}

This work was funded by the British Heart Foundation (grants PG/07/074/23445 and FS/07/005). 
References 


\section{Figure legends}

Fig. 1. Regulation of the cardiomyocyte transcriptome by IL1 $\beta$ vs IL33. Cardiomyocytes were exposed to IL1 $\beta$ or IL33 and the transcriptome analysed with Affymetrix microarrays. Transcripts significantly changed $(>1.5$-fold; FDR $<0.05)$ by either ligand were clustered according to regulation by only IL1 $\beta$ (A) or IL33 (C), or either agonist (B). Heatmaps are normalised to unstimulated controls ( -2.5 (cyan) through 0 (black) to +2.5 (red); $\log _{2}$ scale) and are means for $\mathrm{n}=3$. Clustering on entities used a Euclidean distance matrix and centroid linkage ratio. D-I, qPCR validation of microarray data for Csf1 (D), Csf2 (E), Csf3 (F), Ptgs2 (G), IL1 $\alpha(\mathrm{H})$ and IL $\beta$ (I). Left panels, Cardiomyocytes were exposed to IL1 $\beta$ (solid circles, solid lines) or IL33 (open circles, dashed lines) for the times indicated and mRNA expressions analysed by qPCR. Results are means $\pm \operatorname{SEM}(n=3$ or 4$) .{ }^{*} \mathrm{p}<0.05$ for IL1 $\beta$ relative to zero time, \# $\mathrm{p}<0.05$ for IL33 relative to zero time (repeated measures one-way ANOVA with SNK post-test). Right panels, Microarray data for each mRNA studied are presented relative to unstimulated controls. Results are means for $n=3$.

Fig. 2. Classification of IL1 $\beta$ and IL33-responsive transcripts. IL1 $\beta$-responsive mRNAs identified by microarray analysis were clustered according to the time to maximal upregulation (A) or downregulation (B). Clusters were interrogated for IL33-responsive RNAs. Results are means \pm SEM for each group. ${ }^{*} \mathrm{p}<0.05$ for IL1 $\beta$ relative to zero time, \# $\mathrm{p}<0.05$ for IL33 relative to zero time, $\uparrow \mathrm{p}<0.05$ for IL33a (repeated measures one-way ANOVA with SNK post-test). C, Panther classification of responsive genes. 
[1] Arend WP, Palmer G, Gabay C. IL-1, IL-18, and IL-33 families of cytokines. Immunol Rev 2008;223:20-38.

[2] Lingel A, Weiss TM, Niebuhr M, Pan B, Appleton BA, Wiesmann C et al. Structure of IL-33 and its interaction with the ST2 and IL-1RAcP receptors--insight into heterotrimeric IL-1 signaling complexes. Structure 2009;17:1398-410.

[3] Clerk A, Cullingford TE, Fuller SJ, Giraldo A, Markou T, Pikkarainen S et al. Signaling pathways mediating cardiac myocyte gene expression in physiological and stress responses. $\mathbf{J}$ Cell Physiol 2007;212:311-22.

[4] Netea MG, Simon A, van d, V, Kullberg BJ, van der Meer JW, Joosten LA. IL-1beta processing in host defense: beyond the inflammasomes. PLoS Pathog 2010;6:e1000661. [5] Palmer G, Gabay C. Interleukin-33 biology with potential insights into human diseases. Nat Rev Rheumatol 2011;7:321-9.

[6] Yndestad A, Dama JK, Oie E, Ueland T, Gullestad L, Aukrust P. Role of inflammation in the progression of heart failure. Curr Cardiol Rep 2007;9:236-41.

[7] Kakkar R, Lee RT. The IL-33/ST2 pathway: therapeutic target and novel biomarker. Nat Rev Drug Discov 2008;7:827-40.

[8] Barksby HE, Lea SR, Preshaw PM, Taylor JJ. The expanding family of interleukin-1 cytokines and their role in destructive inflammatory disorders. Clin Exp Immunol 2007;149:217-25.

[9] Kakkar R, Hei H, Dobner S, Lee RT. Interleukin 33 as a mechanically responsive cytokine secreted by living cells. J Biol Chem 2012;287:6941-8.

[10] Sanada S, Hakuno D, Higgins LJ, Schreiter ER, McKenzie ANJ, Lee RT. IL-33 and ST2 comprise a critical biomechanically induced and cardioprotective signaling system. J Clin Invest 2007;117:1538-49. 
[11] Yndestad A, Marshall AK, Hodgkinson JD, Tham eL, Sugden PH, Clerk A. Modulation of interleukin signalling and gene expression in cardiac myocytes by endothelin-1. Int $\mathbf{J}$ Biochem Cell Biol 2010;42:263-72.

[12] Clerk A, Harrison JG, Long CS, Sugden PH. Pro-inflammatory cytokines stimulate mitogen-activated protein kinases, increase phosphorylation of c-Jun and ATF2 and upregulate c-Jun protein in neonatal rat ventricular myocytes. J Mol Cell Cardiol 1999;31:2087-99.

[13] Vaudry D, Lazarovici P, Eiden LE. Signaling pathways for PC12 cell differentiation: making the right connections. Science 2002;296:1648-9.

[14] Marshall AK, Barrett OP, Cullingford TE, Shanmugasundram A, Sugden PH, Clerk A. ERK1/2 signaling dominates over RhoA signaling in regulating early changes in RNA expression induced by endothelin-1 in neonatal rat cardiomyocytes. PLoS One 2010;5:e10027.

[15] Mi H, Dong Q, Muruganujan A, Gaudet P, Lewis S, Thomas PD. PANTHER version 7: improved phylogenetic trees, orthologs and collaboration with the Gene Ontology Consortium. Nucleic Acids Res 2010;38:D204-D210.

[16] Bugaisky L, Zak R. Differentiation of adult rat cardiac myocytes in cell culture. Circ Res 1989;64:493-501.

[17] Zhou YY, Wang SQ, Zhu WZ, Chruscinski A, Kobilka BK, Ziman B et al. Culture and adenoviral infection of adult mouse cardiac myocytes: methods for cellular genetic physiology. Am J Physiol Heart Circ Physiol 2000;279:H429-H436.

[18] Schmitz J, Owyang A, Oldham E, Song Y, Murphy E, McClanahan TK et al. IL-33, an interleukin-1-like cytokine that signals via the IL-1 receptor-related protein ST2 and induces T helper type 2-associated cytokines. Immunity 2005;23:479-90.

[19] Rallidis LS, Zolindaki MG, Pentzeridis PC, Poulopoulos KP, Velissaridou AH, 
Apostolou TS. Raised concentrations of macrophage colony stimulating factor in severe unstable angina beyond the acute phase are strongly predictive of long term outcome. Heart 2004;90:25-9.

[20] Parissis J, Filippatos G, Adamopoulos S, Li X, Kremastinos DT, Uhal BD.

Hematopoietic colony stimulating factors in cardiovascular and pulmonary remodeling: promoters or inhibitors? Curr Pharm Des 2006;12:2689-99.

[21] Streicher JM, Wang Y. The role of COX-2 in heart pathology. Cardiovasc Hematol Agents Med Chem 2008;6:69-79. 